\title{
Spin transport in the Quantum Spin Liquid State in the $S=1$ Kitaev model: role of the fractionalized quasiparticles
}

\author{
Akihisa Koga $^{1}$, Tetsuya Minakawa ${ }^{1}$, Yuta Murakami ${ }^{1}$, and Joji Nasu ${ }^{2}$ \\ ${ }^{1}$ Department of Physics, Tokyo Institute of Technology, Meguro, Tokyo 152- 8551, Japan \\ ${ }^{2}$ Department of Physics, Yokohama National University, Hodogaya, Yokohama 240-8501, Japan
}

\begin{abstract}
We investigate the real-time spin response of the $S=1 \mathrm{Kitaev}$ model upon stimuli of a pulsed magnetic field in the one of the edges using the exact diagonalization method. It is found that the pulsed magnetic field has no effect on the appearance of the spin moments in the quantum spin liquid region, but induces the spin oscillations in the other edge region with a small magnetic field. This is understood by the existence of the itinerant quasiparticles, which carries the spin excitations without the spin polarization in the quantum spin liquid state. This suggests that the spin fractionalizations occur in the $S=1 \mathrm{Kitaev}$ model as well as the exactly solvable $S=1 / 2 \mathrm{Kitaev}$ one and the fractionalized quasiparticles play an essential role in the spin transport.
\end{abstract}

The Kitaev model has attracted much interest since the proposal of the quantum spin model by A. Kitaev ${ }^{1)}$ and suggestion of its implementation in real materials. ${ }^{2)}$ This model is composed of direction-dependent Ising exchange interactions on a honeycomb lattice, which is exactly solvable and its ground state is a quantum spin liquid (QSL) with short-range spin correlations. In this model, quantum spins are fractionalized into the localized and itinerant Majorana fermions due to the quantum many-body effect. ${ }^{1,3-5)}$ The Majorana fermions have been observed recently as a half-quantized plateau in the thermal quantum Hall experiments in the candidate material $\alpha$ - $\mathrm{RuCl}_{3}{ }^{6-10)}$ Furthermore, it is theoretically clarified that distinct energy scales ascribed to the fractionalization appear in the thermodynamic properties such as a double-peak structure in the specific heat, ${ }^{11,12)}$ which stimulates further theoretical and experimental investigations on the spin fractionalization. ${ }^{13-18)}$ Recently, the generalization of the Kitaev model with arbitrary spins ${ }^{19)}$ has been studied, ${ }^{20-29)}$ where similar double peaks in the specific heat have been reported. ${ }^{21)}$ Therefore, the spin fractionalizations are naively expected even in the spin- $S$ Kitaev model although it is no longer solvable.

In our previous manuscipt, ${ }^{30)}$ we have studied the real-time dynamics of the $S=1 / 2 \mathrm{Kitaev}$ model by means of the Majorana mean-field theory. It has been found that, even in the Kitaev QSL with extremely short-ranged spin correlations, the spin excitation propagates in the bulk without spin polarization. This suggests that the spin transport is not caused by the change of local magnetization, but is mediated by the itinerant Majorana fermions. Therefore, the real-time simulation for the spin transport is one of the promising approaches to examine the existence of the itinerant quasiparticles in the spin- $S$ Kitaev model.

In this paper, we investigate the real-time dynamics of the $S=1$ Kitaev model on the honeycomb lattice with two edges by means of the exact diagonalization method. We demonstrate that after the pulsed magnetic field is applied to one of the edges, the oscillation of spin moments does not appear in the bulk, but is induced in the other edge region under the small magnetic field. These results are essentially the same as those in the $S=1 / 2 \mathrm{Kitaev}$ model discussed in our previous paper. ${ }^{30)}$ Therefore, our results support the existence of the spin fractionalization in the $S=1$ Kitaev model.

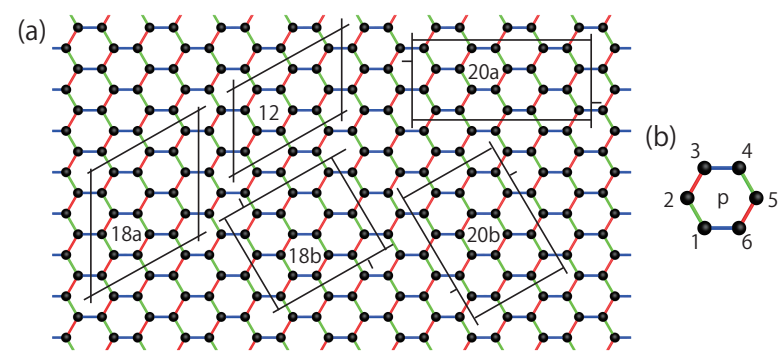

Fig. 1. (a) $S=1 \mathrm{Kitaev}$ model on the honeycomb lattice. Red, blue, and green lines represent the $x, y$, and $z$ bonds, respectively. Clusters used in the exact diagonalizations are presented by the black lines. (b) Plaquette with sites marked 1-6 shown for the local operator $W_{p}$.

Let us consider the $S=1 \mathrm{Kitaev}$ model on the honeycomb lattice, which should be described by the Hamiltonian as

$$
\begin{aligned}
\mathcal{H} & =\mathcal{H}_{0}+\mathcal{H}^{\prime}, \\
\mathcal{H}_{0} & =-J \sum_{\langle i, j\rangle_{x}} S_{i}^{x} S_{j}^{x}-J \sum_{\langle i, j\rangle_{y}} S_{i}^{y} S_{j}^{y}-J \sum_{\langle i, j\rangle_{z}} S_{i}^{z} S_{j}^{z}, \\
\mathcal{H}^{\prime} & =-\sum_{i} h_{i} S_{i}^{z},
\end{aligned}
$$

where $S_{i}^{\gamma}$ is a $\gamma(=x, y, z)$ component of an $S=1$ spin operator at the $i$ th site in the honeycomb lattice. $J$ is the ferromagnetic exchange between the nearest-neighbor spin pairs $\langle i j\rangle_{\gamma}$ on the $\gamma$-bond, and $h_{i}$ is the site-dependent magnetic field applied in the $z$-direction. The model is schematically shown in Fig. 1(a).

We note that the Hamiltonian Eq. (1) has a parity symmetry. This is clearly found if one considers the conventional local basis sets $|m\rangle$ with $m=-1,0,1$, which are the eigenstates of the $S^{z}$. The interactions $S_{i}^{x} S_{j}^{x}$ and $S_{i}^{y} S_{j}^{y}$ inclement or declement of both $m_{i}$ and $m_{j}$, while $S_{i}^{z} S_{j}^{z}$ and $S_{i}^{z}$ do not change them. Therefore, the Hamiltonian Eq. (1) has a global parity symmetry for $S_{t o t}^{z}\left(=\sum_{i} S_{i}^{z}\right)$. In other words, the operator $P_{z}=\exp \left[i \pi S_{\text {tot }}^{z}\right]$ commutes with the Hamiltonian. This leads to the absence of the magnetization in $y$ and $z$ directions 
for any sites, $\left\langle S_{i}^{x}\right\rangle=\left\langle S_{i}^{y}\right\rangle=0$ since $S_{i}^{x}$ and $S_{i}^{y}$ change the parity. Then, in the system, the magnetization appears only in the $z$-direction.

When no mangetic field is applied $\left(h_{i}=0\right)$, the groundstate and finite-temperature properties in the $S=1 \mathrm{Kitaev}$ model has been discussed so far. ${ }^{19-29)}$ In the case, the Kitaev model has the local $Z_{2}$ symmetry on each plaquette. The corresponding operator ${ }^{19,24)}$ is given as,

$$
W_{p}=\exp \left[i \pi\left(S_{1}^{x}+S_{2}^{y}+S_{3}^{z}+S_{4}^{x}+S_{5}^{y}+S_{6}^{z}\right)\right],
$$

where site indexes $1,2, \cdots, 6$ are introduced for a plaquette $p$, as shown in Fig. 1(b). This operator satisfies $W_{p}^{2}=1$ and $\left[\mathcal{H}_{0}, W_{p}\right]=0$. Furthermore, $W_{p}$ commutes with $W_{q}$ on any plaquettes $q$. Therefore, the Hilbert space of the Hamiltonian $\mathcal{H}_{0}$ can be classified into each subspace $\mathcal{S}\left[\left\{w_{p}\right\}\right]$ specified by the set of $w_{p}(= \pm 1)$, which is the eigenvalue of $W_{p}$. When a state is in a certain subspace as $|\psi\rangle=\left|\psi ;\left\{w_{p}\right\}\right\rangle$, the expectation value of the spin operator at the $i$ th site vanishes as $\left\langle\psi\left|S_{i}^{\gamma}\right| \psi\right\rangle=0$. This can be proved when there exists a plaquette satisfying the anticommutation relation $\left\{S_{i}^{\gamma}, W_{p}\right\}=$ 0. In fact, $\left\langle\psi\left|\left\{S_{i}^{\gamma}, W_{p}\right\}\right| \psi\right\rangle=\left\langle\psi\left|S_{i}^{\gamma} W_{p}\right| \psi\right\rangle+\left\langle\psi\left|W_{p} S_{i}^{\gamma}\right| \psi\right\rangle=$ $2 w_{p}\left\langle\psi\left|S_{i}^{\gamma}\right| \psi\right\rangle=0$. Furthermore, examining $\left\langle\psi\left|\left\{S_{i}^{\gamma}, W_{p}\right\} S_{j}^{\gamma}\right| \psi\right\rangle$, one obtains $\left\langle\psi\left|S_{i}^{\gamma} S_{j}^{\gamma}\right| \psi\right\rangle=0$ except for the case with sites $i$ and $j$ located on the same $\gamma$ bond. Therefore, the existence of the local conserved quantity guarantees that the ground state of the $S=1$ Kitaev model with $h=0$ is the quantum spin liquid state with extremely short-ranged spin-spin correlations. ${ }^{19)}$

This discussion may be applicable in the original Hamiltonian $\mathcal{H}$ with nonuniform magnetic field $h_{i}$. When no magnetic field is applied to $i(=1,2,4,5)$ th sites for a plaquette $p$ in Fig. $1(\mathrm{~b}),\left[\mathcal{H}, W_{p}\right]=0$. Then, the Hilbert space is classified by eigenvalues $w_{p}$ in $\mathcal{P}$, where $\mathcal{P}$ stands for the set of the plaquettes satisfying such commutation relations. Then, we obtain $\left\langle S_{i}^{z}\right\rangle=0$ at the $i(=1,2,4,5)$ th sites in the plaquette $p$ in $\mathcal{P}$ since $\left\{S_{i}^{z}, W_{p}\right\}=0$. On the other hand, as for the plaquettes not belonging to $\mathcal{P}$, the corresponding operators do not commute with the Hamiltonian due to the presence of the magnetic field and one cannot prove the absence of the spin moments at the site $1,2,4$, and 5 . If the number of plaquettes not belonging to $\mathcal{P}$ is nonzero, the correlation function between corresponding spins can be finite in general. This results from the lack of the local $Z_{2}$ symmetry in the Kitaev model, and thereby it is highly nontrivial whether or not correlations indeed exist between spins, in particular, even when these spins are separated by the quantum spin liquid region with extremely short-ranged spin-spin correlations.

In our paper, to discuss spin-spin correlations in the Kitaev model, we examine spin transport in the system, where the QSL region is present between the regions under the magnetic field [see Fig. 3(a)]. Before showing the results, we briefly examine how the uniform magnetic field $h\left(=h_{i}\right)$ affects the $S=1 \mathrm{Kitaev}$ model in the bulk. By making use of the exact diagonalization for some clusters with the periodic boundary condition (see Fig. 1), we calculate the magnetization $m_{i}^{z}\left(=\left\langle S_{i}^{z}\right\rangle\right)$, as shown in Fig. 2. When $h=0$, the QSL ground state is realized with $m^{z}=0$. Switching on the magnetic field, the magnetic moment is immediately induced, as shown in Fig. 2. Around $h_{c} / J \sim 0.02$, the magnetization rapidly increases. In the region, the large system size dependence is observed. This appears to be consistent with the results with

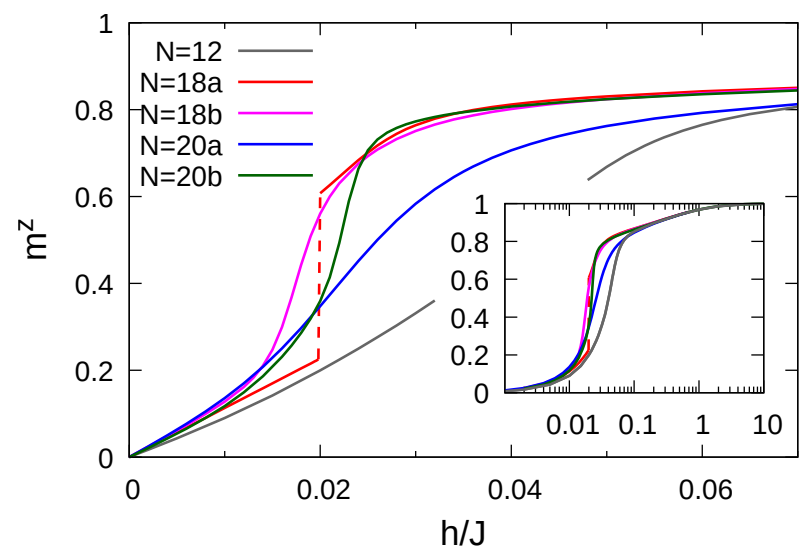

Fig. 2. Magnetization process in the $S=1 \mathrm{Kitaev}$ systems with $N=$ $12,18 a, 18 b, 20 a$, and $20 b$. The vertical dashed line represents the jump singularity of $m^{z}$ in the $18 a$ cluster.

$h_{c} \sim 0.01 J$ in the (111)-direction magnetic field. ${ }^{26,28,29)}$ On the other hand, we have confirmed that the ground state always belongs to the subspace with even parity, including the results in the $N=18 a$ cluster with a jump singularity in the magnetization process. Therefore, it is still unclear whether or not the phase transition occurs to the polarized state in the thermodynamic limit. We also note that these results for the $S=1$ system are similar to those in the $S=1 / 2 \mathrm{Kitaev}$ model, where the phase transition occurs to the polarized state at $h / J \sim 0.042$ within the mean-field theory. ${ }^{31)}$ Therefore, we believe that there exists the energy scale characteristic of the spin excitations in the $S=1 \mathrm{Kitaev}$ model. In the following, we deal with the system with a tiny magnetic field $\left(<h_{c}\right)$ to discuss the existence of the spin fractionalization in the $S=1$ Kitaev model.

To study the spin transport in the $S=1 \mathrm{Kitaev}$ model, we consider the site-dependent magnetic field, which is defined as

$$
h_{i}=\left\{\begin{array}{ll}
h_{L}(t) & i \in \mathrm{L} \\
0 & i \in \mathrm{B} \\
h_{R} & i \in \mathrm{R}
\end{array},\right.
$$

where $h_{L}\left(h_{R}\right)$ is the time-dependent (static) magnetic field applied to the left $(\mathrm{L})$ [right $(\mathrm{R})$ ] region. In the bulk (B) region, no magnetic field is applied, and the QSL state is always realized without the magnetization. The system is schematically shown in Fig. 3(a).

We explain the outline of our real-time simulations by means of the exact diagonalization. The initial ground state $|\psi\rangle$ at $t \rightarrow-\infty$ is obtained by means of the Lanczos and inverse iteration methods. The time-evolution of the wave function is calculated by the time-dependent Schrödinger equation as,

$$
i \frac{d}{d t}|\psi(t)\rangle=\mathcal{H}(t)|\psi(t)\rangle
$$

Then, we compute the magnetization and nearest-neighbor spin-spin correlation on the $\gamma$-bond, which are given as

$$
\begin{aligned}
m_{i}^{z}(t) & =\left\langle\psi(t)\left|S_{i}^{z}\right| \psi(t)\right\rangle, \\
C_{i j}(t) & =\left\langle\psi(t)\left|S_{i}^{\gamma} S_{j}^{\gamma}\right| \psi(t)\right\rangle .
\end{aligned}
$$

In this study, we introduce the pulsed magnetic field in the $\mathrm{L}$ 


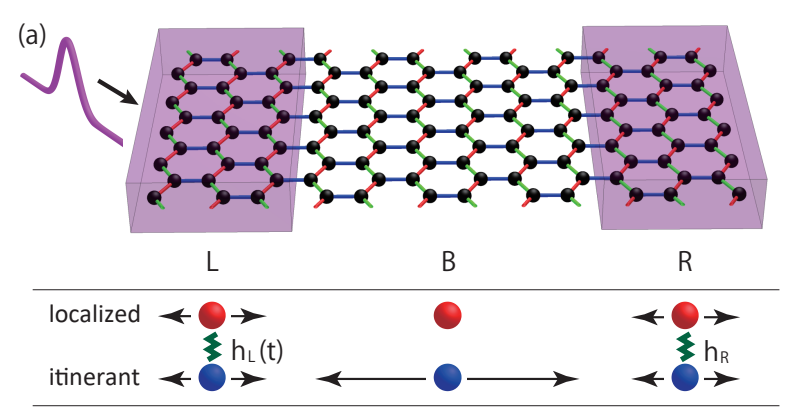

(b)

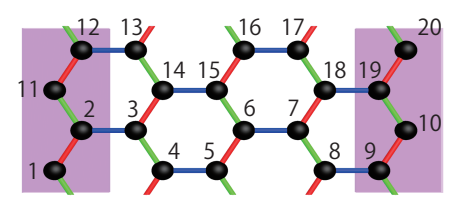

Fig. 3. (a) Schematic picture of the Kitaev system on the honeycomb lattice with two edges. The lattice is composed of three regions. The static magnetic field $h_{R}$ is applied in the right (R) region, where the magnetization appears. In the bulk (B) region, no magnetic field is applied and the QSL state is realized without the magnetization. Time-dependent pulsed magnetic field is introduced in the left (L) region. (b) 20-site cluster used in the exact diagonalization. The numbers represent the index of the lattice site.

region, which is explicitly given as

$$
h_{L}(t)=\frac{A}{\sqrt{2 \pi \sigma^{2}}} \exp \left[-\frac{t^{2}}{2 \sigma^{2}}\right],
$$

where $A$ and $\sigma$ are magnitude and width of the Gaussian pulse. In the following, we fix $A=1$ and $\sigma=2 / J$.

In this calculation, we examine the 20 -site cluster with two edges, where the periodic boundary condition is imposed for the direction perpendicular to the edge, as shown in Fig. 3(b). In the cluster, the $\mathrm{R}$ and $\mathrm{L}$ regions include four sites. There exist twelve sites in the B region, where no magnetic field is applied. Although the cluster we treat is too small, the spin transport characteristic of the Kitaev system is expected to be captured since there exist four plaquettes with the local $Z_{2}$ symmetry, which is crucial for the peculiar spin transport, in the $B$ region. In fact, we have confirmed that, in the initial state $(t \rightarrow-\infty)$, the site-dependent magnetization $m_{i}^{z}$ appears only in the R region $\left(m_{9}^{z}=m_{19}^{z}=0.217\right.$ and $\left.m_{10}^{z}=m_{20}^{z}=0.220\right)$. Figure 4 shows the time dependence of the change in the spin moment $\Delta m_{i}^{z}\left[=m_{i}^{z}(t)-m_{i}^{z}(-\infty)\right]$ after the pulsed magnetic field is introduced. The magnetic moments at the sites 1 and 2 in the $\mathrm{L}$ region are induced by the pulsed magnetic field. On the other hand, no magnetic moment appears in the B region, which is consistent with the existence of the local conserved quantity. On the other hand, after some delay, the spin oscillation is induced at the sites 9 and 10 in the R region. This means that the wave-packet triggered by the magnetic pulse in the $\mathrm{L}$ region reaches the $\mathrm{R}$ region through the $\mathrm{B}$ region without spin oscillations. Since $m_{i}^{x}(t)=m_{i}^{y}(t)=0$ for any sites, we can say that the spin moment plays no role in the spin transport in the $S=1 \mathrm{Kitaev}$ model. This remarkable phenomenon is similar to that in the $S=1 / 2 \mathrm{Kitaev}$ model, ${ }^{30}$ ) where the spin transport is mediated by the itinerant Majorana fermions. Therefore, our results suggest the existence of the itinerant quasiparticles, which is not associated with the spin

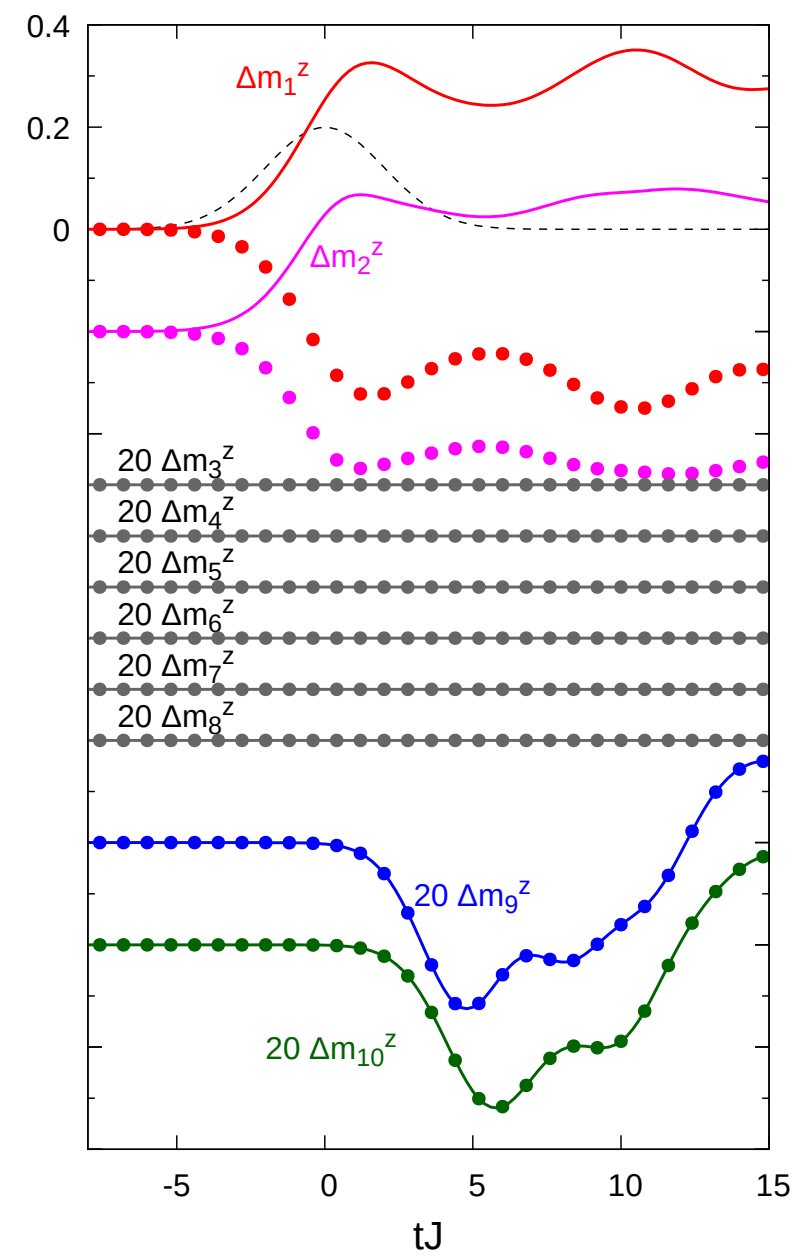

Fig. 4. Real-time evolution of the change in the local magnetization in the system with $h_{R} / J=0.01$ after the introduction of the pulsed magnetic field with $A=1$ and $\sigma=2 / J$ shown as the dashed line (offset for clarity). Dotted lines represent the results for the pulse with $A=-1$ and $\sigma=2 / J$.

polarization even in the $S=1 \mathrm{Kitaev}$ model. Namely, we expect that in the $S=1 \mathrm{Kitaev}$ model without a static magnetic field, the spin degree of freedom is fractionalized into two; itinerant and localized quasiparticles owing to the existence of the local $Z_{2}$ symmetry. The pulsed magnetic field in the $\mathrm{L}$ region creates the itinerant and localized quasiparticle excitations, while only the formers propagate in the whole system. In the $\mathrm{R}$ region, the itinerant and localized quasiparticles are hybridized by the static magnetic field due to the lack of the local $Z_{2}$ symmetry, leading to the finite spin oscillations. The scenario for the spin transport is schematically shown in Fig. 3(a).

The spin fractionalization in the $S=1$ Kitaev model has been suggested in the thermodynamic properties such as a double-peak structure in the specific heat. ${ }^{21)}$ We note that the higher temperature peak is closely related to the nearestneighbor spin-spin correlations $C_{i j}$. It is known that the higher temperature peak in the $S=1 / 2$ case corresponds to the motion of the itinerant Majorana fermions. Therefore, one can expect that the flow of $C_{i j}$ is regarded as the motion of the itinerant quasiparticles in the $S=1 \mathrm{Kitaev}$ model. Figures 5(a), 5(b) and 5(c) show the real-time evolution of the change in the nearest-neighbor spin correlations on the $x$-, $y$-, and $z$-bonds except for the $\mathrm{L}$ region. Oscillations appear in the spin-spin 

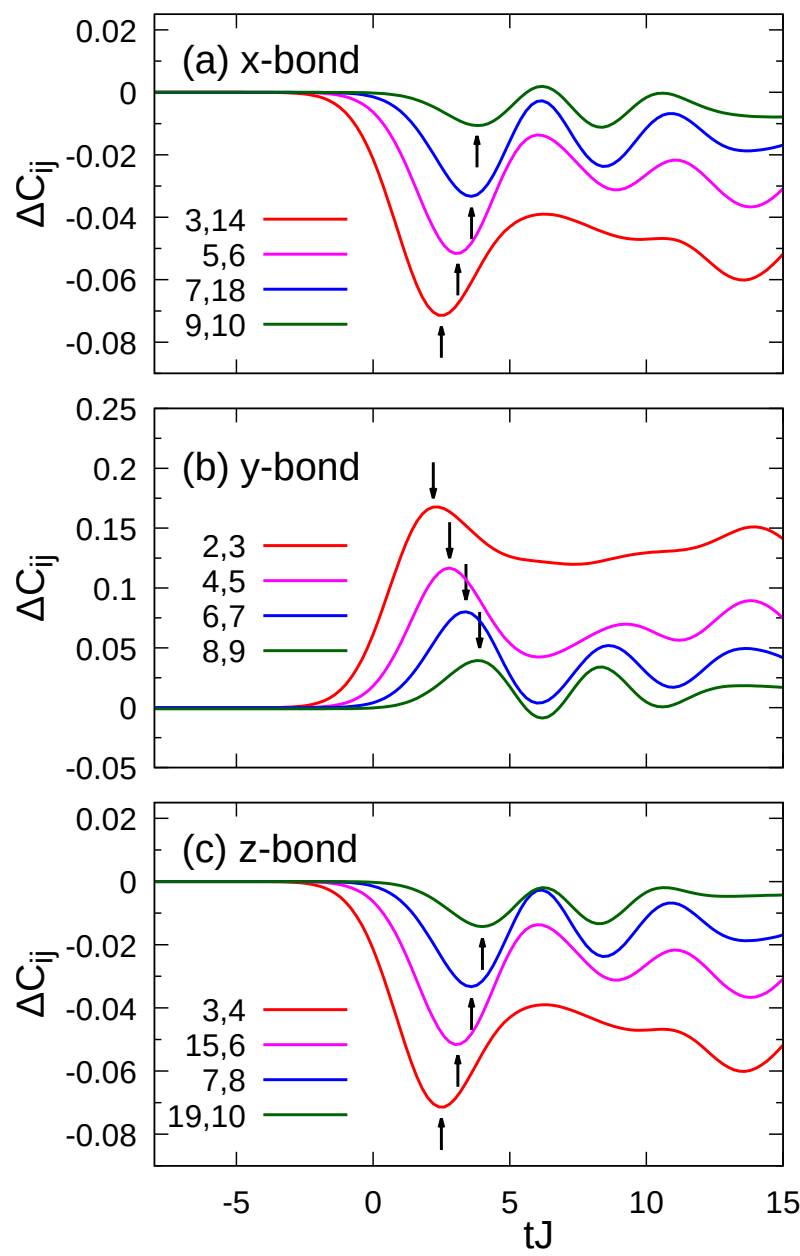

Fig. 5. Real-time evolution of the change in the nearest-neighbor correlations on the (a) $x$-bond, (b) $y$-bond, and (c) $z$-bond in the B and $\mathrm{R}$ region of the $S=1$ Kitaev system with $h_{R} / J=0.01, A=1$, and $\sigma=2 / J$. Pairs of the numbers indicate two sites coupled by the Kitaev exchanges in the 20 -site cluster shown in Fig. 3.

correlations on all exchanges even in the B region. We also find that the change of the quantities becomes small with increasing the distance from the $\mathrm{L}$ region. Associated with this change, the characteristic time, where $\left|\Delta C_{i j}\right|$ takes a first maximal value (shown as arrows in Fig. 5), becomes longer. This implies that the wave-packet created by the pulsed magnetic field at the $\mathrm{L}$ region indeed flows to the $\mathrm{R}$ region through the $\mathrm{B}$ region. The second maximal values are considered to be caused by the reflection of the flow at the right edge since the peaks shift to the left side as time elapses.

We also note the interesting pulse-field dependence of the phenomena originated from the $Z_{2}$ symmetry. ${ }^{30)}$ The system has the local $Z_{2}$ symmetry in the $L$ region before the pulsed magnetic field is applied. In this case, each eigenstate is specified by a set of the eigenvalues of $W_{p}$ in the L region. The Hamiltonian for the magnetic pulse has the offdiagonal elements between distinct subspaces. An important point is that the operator $S_{i}^{z}$, in general, flips two eigenvalues of $W_{p}$ for adjacent plaquettes sharing the same $z$-bond, which connects the $i$ th site and its pair site. Therefore, if the ground state belongs to the subspace with the configuration $\left\{w_{p}\right\}$, only even-order perturbations in the pulsed magnetic field contribute the expectation value for the operator $O$ satisfying $\left[O, W_{p}\right]=0$ with $p \in L$. This means that this expectation value is independent of the sign of the pulsed field. To confirm this, we calculate the time-dependent spin moments after the pulsed magnetic field in the $-z$ direction $(A=-1)$. The results are shown as the dotted lines in Fig. 4. In the L region, the magnetic moments are induced in the direction of the applied field. By contrast, in the $\mathrm{B}$ and $\mathrm{R}$ regions, the results do not depend on the sign of the pulsed magnetic field.

Finally, we briefly comment on the nature of the low-lying excitation in the $S=1 \mathrm{Kitaev}$ system. ${ }^{21,26-29)}$ The real-time simulation, in principle, allows us to clarify if the system is gapped or gapless, by examining the velocity and decay rate of the wave packet created by the pulsed field with small $A$ and/or large $\sigma$. However, in the small-size numerical calculations, it should be hard to evaluate them due to the interference effect for the multiple reflections. Therefore, further numerical calculations for the larger systems are necessary to clarify the elementary excitations in the $S=1 \mathrm{Kitaev}$ model, which is now under consideration.

In summary, we have studied the real-time dynamics of the $S=1$ Kitaev model on the honeycomb lattice. Applying the pulsed magnetic field to one of the edges in the system, spin oscillations never appear in the bulk while they appear in the other edges. Similar behavior appears in the $S=1 / 2$ Kitaev model, where fractionalized Majorana fermions flow in the system. Therefore, our results suggest the existence of the spin fractionalization in the $S=1 \mathrm{Kitaev}$ model and the spin transport is mediated by the fractionalized itinerant quasiparticles. This behavior should be common in the spin- $S$ Kitaev model, which is consistent with thermodynamic properties such as the double-peak structure in the specific heat and the half-plateau in the entropy. ${ }^{21)}$ It is also important to clarify the spin transport in the spin- $S$ Kitaev models while finite temperature calculations suggest that the entropy of the quantum spins is split in half into those of the itinerant and localized quasiparticles. These interesting problems remain as future issues.

Acknowledgments Parts of the numerical calculations are performed in the supercomputing systems in ISSP, the University of Tokyo. This work was supported by Grant-in-Aid for Scientific Research from JSPS, KAKENHI Grant Nos. JP19K23425 (Y.M.), JP19H05821, JP18K04678, JP17K05536 (A.K.), JP16H02206, JP18H04223, JP19K03742 (J.N.), by JST CREST (JPMJCR1901) (Y.M.), and by JST PREST (JPMJPR19L5) (J.N.).

1) A. Kitaev: Ann. Phys. (N. Y.) 321 (2006) 2.

2) G. Jackeli and G. Khaliullin: Phys. Rev. Lett. 102 (2009) 017205.

3) X.-Y. Feng, G.-M. Zhang, and T. Xiang: Phys. Rev. Lett. 98 (2007) 087204.

4) H.-D. Chen and J. Hu: Phys. Rev. B 76 (2007) 193101.

5) H.-D. Chen and Z. Nussinov: J. Phys. A: Math. Theor. 41 (2008) 075001 .

6) K. W. Plumb, J. P. Clancy, L. J. Sandilands, V. V. Shankar, Y. F. Hu, K. S. Burch, H.-Y. Kee, and Y.-J. Kim: Phys. Rev. B 90 (2014) 041112.

7) Y. Kubota, H. Tanaka, T. Ono, Y. Narumi, and K. Kindo: Phys. Rev. B 91 (2015) 094422.

8) J. A. Sears, M. Songvilay, K. W. Plumb, J. P. Clancy, Y. Qiu, Y. Zhao, D. Parshall, and Y.-J. Kim: Phys. Rev. B 91 (2015) 144420.

9) M. Majumder, M. Schmidt, H. Rosner, A. A. Tsirlin, H. Yasuoka, and M. Baenitz: Phys. Rev. B 91 (2015) 180401.

10) Y. Kasahara, T. Ohnishi, Y. Mizukami, O. Tanaka, S. Ma, K. Sugii, N. Kurita, H. Tanaka, J. Nasu, Y. Motome, T. Shibauchi, and Y. Matsuda: Nature 559 (2018) 227. 
11) J. Nasu, M. Udagawa, and Y. Motome: Phys. Rev. B 92 (2015) 115122.

12) J. Nasu, J. Yoshitake, and Y. Motome: Phys. Rev. Lett. 119 (2017) 127204.

13) J. Chaloupka, G. Jackeli, and G. Khaliullin: Phys. Rev. Lett. 105 (2010) 027204.

14) Y. Yamaji, Y. Nomura, M. Kurita, R. Arita, and M. Imada: Phys. Rev. Lett. 113 (2014) 107201.

15) V. M. Katukuri, S. Nishimoto, V. Yushankhai, A. Stoyanova, H. Kandpal, S. Choi, R. Coldea, I. Rousochatzakis, L. Hozoi, and J. v. d. Brink: New J. Phys. 16 (2014) 013056.

16) T. Suzuki, T. Yamada, Y. Yamaji, and S.-i. Suga: Phys. Rev. B 92 (2015) 184411.

17) Y. Yamaji, T. Suzuki, T. Yamada, S.-i. Suga, N. Kawashima, and M. Imada: Phys. Rev. B 93 (2016) 174425.

18) Y. Kato, Y. Kamiya, J. Nasu, and Y. Motome: Phys. Rev. B 96 (2017) 174409.

19) G. Baskaran, D. Sen, and R. Shankar: Phys. Rev. B 78 (2008) 115116.
20) T. Suzuki and Y. Yamaji: Phys. B: Condense. Matter 536 (2017) 637.

21) A. Koga, H. Tomishige, and J. Nasu: J. Phys. Soc. Jpn. 87 (2018) 063703.

22) J. Oitmaa, A. Koga, and R. R. P. Singh: Phys. Rev. B 98 (2018) 214404.

23) T. Minakawa, J. Nasu, and A. Koga: Phys. Rev. B 99 (2019) 104408.

24) A. Koga and J. Nasu: Phys. Rev. B 100 (2019) 100404.

25) P. P. Stavropoulos, D. Pereira, and H.-Y. Kee: Phys. Rev. Lett. 123 (2019) 037203.

26) H.-Y. Lee, N. Kawashima, and Y. B. Kim: arXiv p. 1911.07714.

27) X.-Y. Dong and D. N. Sheng: arXiv p. 1911.12854

28) Z. Zhu, Z.-Y. Weng, and D. N. Sheng: arXiv p. 2001.05054.

29) I. Khait, P. P. Stavropoulous, H.-Y. Kee, and Y. B. Kim: arXiv p. 2001.06000 .

30) T. Minakawa, Y. Murakami, A. Koga, and J. Nasu: arXiv (2019) 1912.10599.

31) J. Nasu, Y. Kato, Y. Kamiya, and Y. Motome: Phys. Rev. B 98 (2018) 060416 . 